\title{
AERODYNAMIC PERFORMANCE PREDICTIONS OF SINGLE AND TWIN JET AFTERBODIES
}

\author{
John R. Carlson* \\ S. Paul Pao** \\ NASA Langley Research Center \\ Hampton, VA \\ Khaled S. Abdol-Hamid*** \\ Analytical Services and Materials, Inc. \\ Hampton, VA \\ William T. Jones ${ }^{+}$ \\ Computer Sciences Corporation \\ Hampton, VA
}

\begin{abstract}
$\underline{\text { Abstract }}$
The multiblock three-dimensional Navier-Stokes method PAB3D was utilized by the Component Integration Branch (formerly Propulsion Aerodynamics Branch) at the NASALangley Research Center in an international study sponsored by AGARD Working Group \#17 for the assessment of the state-of-the-art of propulsion-airframe integration testing techniques and CFD prediction technologies. Three test geometries from ONERA involving fundamental flow physics and four geometries from NASA-LaRC involving realistic flow interactions of wing, body, tail, and jet plumes were chosen by the Working Group. An overview of results on four (1 ONERA and $3 \mathrm{LaRC}$ ) of the seven test cases is presented. External static pressures, integrated pressure drag and total drag were calculated for the Langley test cases and jet plume velocity profiles and turbulent viscous stresses were calculated for the ONERA test case. Only selected data from these calculations are presented in this paper. The complete data sets calculated by the participants will be presented in an AGARD summary report. Predicted surface static pressures compared favorably with experimental data for the Langley geometries. Predicted afterbody drag compared well with experiment. Predicted nozzle drag was typically low due to over-compression of the flow near the trailing edge. Total drag was typically high. Predicted jet plume quantities on the ONERA case compared generally well with data.
\end{abstract}

\section{Introduction}

The AGARD Fluid Dynamics Panel sponsored Working Group (WG) \#17 for the investigation of the aerodynamics of 3-D aircraft afterbodies. This group extended the work of Working Group \#8 which reported on the aerodynamics of 2-D afterbodies in 1986, ref. 1. The panel recognized the extremely complex nature of the flow in the aft

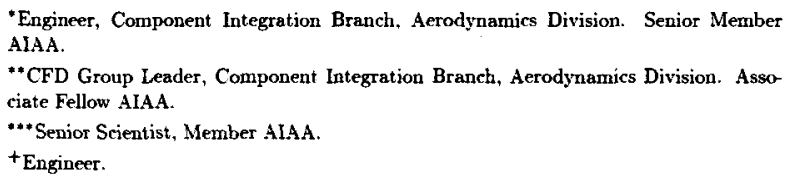

Copyright (c) 1995 by the American Institute of Aeronautics and Astronautics, Inc No copyright is asserted in the United States under Title 17 , U.S. Code. The U.S Government has royalty-free license to exercise all rights under the copyright claimed herein for Governmental purposes. All other rights are reserved by the copyright owner. region of fighter aircraft due to the various interactions of the flows about the fuselage, empennage, and nozzle boattails. These flows can involve such features as separated flow, shock/boundary layer interactions and multiple jet exhaust plumes. The panel, in addition to seeking to develop recommendations for improvements in propulsion testing techniques, sought to continue the evaluation of state-ofthe-art computational methods for their ability in predicting these complex afterbody flows.

The Working Group members selected seven test geometries for the evaluation program. A combination of single and twin-jet configurations were selected emphasizing different aspects of afterbody flows. Three test configurations proposed by ONERA (designated A.x) were chosen for the evaluation of prediction techniques for basic flow physics. These configurations had extensive flow measurement databases such as off-body flow quantity surveys with both hotand cold-jet simulations (A.2). Four Langley test cases (designated B.x) were chosen for being more realistic aerodynamic configurations that would involve complex attached and separated external flows interacting with simulated jet plumes. The data acquired on these models included surface static pressure distributions and force balance data on the model afterbody.

Several issues impair accurate code calibration/validation efforts. Excepting the validity of the computational fluid dynamics (CFD) itself, knowledge of the actual model shape, accuracy of the instrumentation, interference from facility related factors, and the accuracy of setting the facility flow parameters all contribute to uncertainties in the comparisons. Most of the test geometries for the WG\#17 study were well documented. Several of the models were measured after the tests to determine the manufactured shape. All the test cases had well defined free-stream and jet operating conditions.

Highlights of results of the Component Integration Branch (CIB), NASA-LaRC (formerly Propulsion Aerodynamics Branch) involvement in the WG\#17 program are presented. The multiblock three-dimensional Navier-Stokes method PAB3D was used for all the calculations. Jet plume velocity profiles and turbulent viscous stresses were calculated for the A.2 ONERA test case. External static pressures, integrated pressure drag and total drag were calculated for the Langley test cases B.1, B.2 and B.3. Calculations were made on B.4 by CIB but are not presented here. 


\section{Nomenclature}

\begin{tabular}{|c|c|}
\hline$A_{\max }$ & $\begin{array}{l}\text { maximum body cross-sectional area, } \\
0.0273 \mathrm{~m}^{2}\end{array}$ \\
\hline$A_{\text {ref }}$ & wing reference area, $0.4286 \mathrm{~m}^{2}$ \\
\hline$C_{D}$ & $\begin{array}{l}\text { drag coefficient, } \frac{F}{q_{\infty} A_{\max }}(\mathrm{B} .1, \mathrm{~B} .2) \\
\frac{F}{q_{\infty} A_{r e f}} \text { (B.3) }\end{array}$ \\
\hline$C_{p}$ & pressure coefficient, $\frac{p-p_{\infty}}{q_{\infty}}$ \\
\hline$D_{m}$ & body maximum diameter (ONERA A.2), m \\
\hline$F$ & axial force along body axis, $\mathrm{N}$ \\
\hline$l$ & $\begin{array}{l}\text { length of body }(0.18212, B .1, B .2 ; \\
0.17474, B .3), \mathrm{m}\end{array}$ \\
\hline$M, M_{\infty}$ & free-stream Mach number \\
\hline$N P R$ & nozzle pressure ratio, $\frac{p_{t, j}}{p_{\infty}}$ \\
\hline$p$ & static pressure, $\mathrm{Pa}$ \\
\hline$p_{t, j}$ & jet total pressure, $\mathrm{Pa}$ \\
\hline$p_{\infty}$ & free-stream static pressure, $\mathrm{Pa}$ \\
\hline$q_{\infty}$ & free-stream dynamic pressure, $\mathrm{Pa}$ \\
\hline Rey & Reynolds number \\
\hline$T_{t, j}$ & $\begin{array}{l}\text { jet total temperature, (typ. cold jet } \\
300 \mathrm{deg}), \mathrm{K}\end{array}$ \\
\hline$u_{\infty}$ & free-stream velocity, $\mathrm{m} / \mathrm{sec}$ \\
\hline$u$ & axial velocity, $\mathrm{m} / \mathrm{sec}$ \\
\hline$u^{\prime}, w^{\prime}$ & Reynolds stress velocity components, $\mathrm{m} / \mathrm{sec}$ \\
\hline$x$ & axial distance, $\mathrm{m}$ \\
\hline$y$ & lateral distance from model centerline, $m$ \\
\hline$z$ & $\begin{array}{l}\text { vertical or radial distance from model } \\
\text { centerline, } \mathbf{m}\end{array}$ \\
\hline$\alpha$ & flow angle-of-attack, deg \\
\hline$\phi$ & angular location of pressure orifices, deg \\
\hline
\end{tabular}

Subscripts

$\begin{array}{ll}A & \text { afterbody component contribution } \\ C L & \text { centerline } \\ N & \text { nozzle component contribution } \\ P & \text { pressure force contribution } \\ T & \text { total forces from components } \\ t & \text { total conditions }\end{array}$

HT horizontal tail component contribution

VT vertical tail component contribution

\section{Computational Procedure}

\section{Governing Equations}

The code used by CIB at Langley was the general 3-D Navier-Stokes method PAB3D. This code has several computational schemes and different turbulence models that can be utilized, as described in more detail in references 2 and 3 . The governing equations are the Reynolds-averaged simplified Navier-Stokes equations (RANS) obtained by neglect- ing all streamwise derivatives of the viscous terms. The resulting equations are written in generalized coordinates and conservation form. The implementation of the full threedimensional viscous stresses are reduced to thin-layer viscous assumptions, although full Navier-Stokes simulation is an option. The diffusion terms are centrally differenced and the inviscid flux terms are upwind differenced. Two finite volume flux-splitting schemes are used to construct the convective flux terms. The Roe upwind scheme with third order accuracy is used in evaluating the explicit part of the governing equations and the van Leer scheme is used to construct the implicit operator.

The user will typically utilize the Roe scheme procedure to sweep streamwise through the computational domain and the van Leer scheme for the solution of the cross-plane (i.e., $i=$ constant) of a three dimensional problem. A single-cell wide, two-dimensional mesh defined with the $i$ direction of the grid oriented in the conventional streamwise direction will converge more slowly using the Roe relaxation solution scheme compared to solving the equivalent problem with the van Leer scheme. Therefore the $i$ and $j$ directions of the 2-D mesh are swapped allowing the entire flowfield to be solved implicitly with each iteration. The explicit sweep is not used since only one cell exists in the $i$ direction. The implicit scheme has the potential of a much higher rate of convergence and typically provides a solution in less time.

Several near-wall models and compressibility corrections are available to be used with several formulations of linear two-equation k- $\epsilon$ turbulence model, (e.g. Standard, Jones \& Launder, Yang \& Shih). The $\mathrm{k}-\epsilon$ turbulence model equations are uncoupled from the RANS equations and can be solved with a different time step than that of the principle flow solution. The latest version of the code (V.13) has the capability to provide Algebraic Reynold's Stress turbulence simulations, though this was not implemented in the code at the time of this study. All flow solutions were developed with PAB3D-V10 using the Jones \& Launder high Reynolds number formulation of $k-\epsilon$. The jet plume turbulence simulation also utilized the high-Reynolds number formulation with the compressible dissipation of Sarkar, ref. 4. The damping function of Launder and Sharma was used to control the near-wall behavior of $k$ and $\epsilon$ was set to zero at solid surfaces, ref. 5 .

\section{Solution Process}

The calculation of laminar flow solutions require the memory usage of 15 words per grid point. Turbulent flow solutions using the linear two-equation $\mathrm{k}-\epsilon$ model requires 20 words per grid point. The code speed was $43 \mu \mathrm{sec}$ per grid point (Cray 2 time) for solving turbulent flow simulations.

Several parameters were used to gauge solution convergence for the A.2.2 case. Afterbody drag and nozzle mass flow, calculated by the performance package, ref. 6 , and solution residual were used to determine the solution status at the coarse, medium, and fine grid levels. Typically mass flow variance of less than 0.1 percent and afterbody drag variance of less than 0.25 percent for several hundred iterations were achieved. Similar indicators for convergence were used for the B.1 cases. External surface static pressure distributions were monitored for an indication of convergence for the B.2 and B. 3 cases. 


\section{Grid Topology and Block Interfaces}

All grids utilized $\mathrm{H}-\mathrm{H}$ or $\mathrm{H}-\mathrm{O}$ type mesh topologies and had block dimensions that were multiples of 4 . The ONERA A.2 case was gridded with a single cell wide 5 degree wedge grid with the streamwise flow direction oriented along the $j$ index to utilize the implicit flow solver in the code for faster solution convergence. The B.1 mesh had the same topology as the A.2 mesh. The conventional grid orientation was used for the Langley B.2 and B.3 cases, i.e. $i$ index, along which the solution relaxation scheme occurs, is oriented in the streamwise direction. Block faces were matched one-to-one or integer-to-one, except for the A.2 case where some block face cell mis-matches occurred for gridding convenience.

The conservative patch interface package of Pao \& AbdolHamid, ref. 7, enables the code to properly transmit information between mis-matched block interfaces. Integer-toone interfaces are considered a subset of the arbitrary block interface and do not need to be specified as such to the patching code. The patching program writes a connectivity database as a preprocessor prior to execution of the flow solver. Each entry to the patch database contains cell face areas and indices relating that cell with all other cells that will share momentum flux information. The database information is automatically re-allocated internal to the code during mesh sequencing. As a result, each block can be sequenced at different levels and the correct interface information is maintained at the cell level. However, it is important to note that features in the flow developed on one side of an interface should not be obliterated on the other side due to too severe a grid density mis-match.

Third-order continuity in transmitting the fluxes across block boundaries is maintained by the code; lower order continuity may be specified by the user if required. Equal cell size spacing on either side of an interface in directions normal to the interface must still be maintained regardless of the mesh sequencing level of the block.

\section{Boundary Conditions}

For this study, solid walls were treated as no-slip adiabatic surfaces. The solid wall boundary condition was satisfied by setting the momentum flux of the solid wall cell face to zero. The boundary conditions used for the internal nozzle flow path were fixed total pressure, total temperature and flow angle at the plenum block inflow face. A boundary condition for the Riemann invariants along the characteristics was specified for the external freestream inflow face and the lateral freestream outer boundary of the flow domain. An extrapolation boundary condition was applied on the downstream outflow face where both the free stream and the nozzle plume exit the computational domain. The axisymmetric flow assumption for the single-cell grids was implemented by placing flow symmetry conditions to the lateral side boundaries of the computational domain.

\section{Results and Discussion}

\section{Test Case A.2}

The A.2 test case was an axisymmetric single-engine model with a $0.105 \mathrm{~m}$ diameter, $0.993 \mathrm{~m}$ length body mounted $0.382 \mathrm{~m}$ from the tunnel wall on a 10 percent thick strut. Data were obtained in the Center. The A.2 case consisted of a cold jet condition (A.2.1) and a hot jet condition (A.2.2). The jet total temperature for A.2.1 was $T_{t_{j}}=300 \mathrm{~K}$ and $935 \mathrm{~K}$ for A.2.2. Both A.2.1 and A.2.2 were tested at free stream conditions of $\mathrm{M}=0.8, p_{t_{\infty}}=100000 \mathrm{~Pa}, T_{t_{x}}=$ $300 \mathrm{~K}$ with the jet operating at an NPR $=4.8$. Experimental data obtained by ONERA include streamwise and axial flow velocities, turbulent kinetic energy and Reynold s stress surveys at several stations downstream of the nozzle exit. Only data from A.2.2 is presented in this paper.

The computational blocking used to simulate the A.2 model is shown in figure 1 . A total of 27 blocks were used for the complete configuration. An in-house algebraic grid generator was used to generate the single-cell axisymmetric grid. The grid included modeling of the free stream in front of the model; the model nose, centerbody, and boattail: and nozzle internal geometries with high grid density in the nozzle trailing edge region. The nozzle trailing edge shape was difficult to accurately define due to uncertainty in the mating of the internal nozzle and external afterbody shells, as well as, some thermal expansion movement due to the hot jet. The original surface geometry definition used was that lofted by McDonnell-Douglas Aerospace in St. Louis, Missouri. A detail of the trailing edge grid is shown in figure 2. The modified definition, figure 3, was developed later in the study after conversations with other program participants. The modified shape appears to be more representative of the actual configuration.

Figure 4 is a comparison of the experimental nozzle boattail surface static pressure distributions with computed data using the two different boattail definitions. Several modifcations to the boattail definition occurred in the process of modifying the trailing edge shape. The transition between two sequential conical sections defining the boattail geometry was sharpened resulting considerably stronger flow acceleration from about $-1.8<x / D_{m}<-0.6$. The terminal boattail angle remained unchanged and as a result the static pressure distribution from $-0.6<x / D_{m}<0$ were very similar for both trailing edge shapes. The over-stagnation of the flow at the nozzle trailing edge is typical of turbulent flow calculations using the Jones \& Launder $\mathrm{k}-\epsilon$ model. In general, the impact of the trailing edge geometry on predicted nozzle boattail static pressure distribution was small, especially when compared to the effect of corner sharpness between the two conical boattail sections.

Figures 5 and 6 are flowfield Mach contours in the vicinity of the nozzle/boattail for the original and modified trailing edge geometry configurations, respectively. Figures 7,8, and 9 are radial distributions of several flowfield quantities at station (survey plane) $x / D_{m}=0.4840$. The vertical dashed lines in figures 5 and 6 are the approximate locations of the survey plane. There were some small detailed changes in the plume which resulted from the t.e. geometry modification. The peak Mach number at the plume centerline increased from $M=2.58\left(x / D_{m}=0.42\right)$ for the original grid to approximately $M=2.84$ for the modified grid due to the stronger expansion from the inner lip of the modified geometry. The comparison of computations with axial velocity LV-survey data at $x / D_{m}=0.4840$, figure 7 , shows excellent overall agreement using either geometry, except near the plume centerline. Figure 8 presents a comparison of predicted axial normal stresses $\left|u^{\prime}\right| / u_{\infty}$ with experimental data. The predicted peak is lower than the data which is 
Table 1. Test Cases

\begin{tabular}{|c|c|c|c|c|c|c|c|c|c|c|c|}
\hline \multicolumn{6}{|c|}{ Identification } & \multicolumn{3}{|c|}{ Free-stream conditions } & \multicolumn{2}{|c|}{ Jet How condition } & \multirow{2}{*}{$\begin{array}{l}\text { Position } \\
a, \text { deg }\end{array}$} \\
\hline Case & $\begin{array}{c}\text { Horiz. } \\
\text { tail }\end{array}$ & $\begin{array}{c}\text { Vert. } \\
\text { tail }\end{array}$ & $\begin{array}{c}\text { \# of } \\
\text { engines }\end{array}$ & $\begin{array}{l}\text { Nozzle } \\
\text { shape }\end{array}$ & $\begin{array}{c}\text { Boattail } \\
\text { separation }\end{array}$ & $M_{\infty}$ & $\begin{array}{l}\text { Nom. } \\
q_{\infty}, \text { psi }\end{array}$ & $\begin{array}{l}\text { Nom. } \\
T_{t_{\infty},}{ }^{\circ} \mathrm{R}\end{array}$ & $N P R$ & $\begin{array}{c}\text { Nom. } \\
T_{t_{j}},{ }^{\circ} \mathrm{R}\end{array}$ & \\
\hline B.1.1 & None & None & One & Axi. & Yes & 0.901 & 4.94 & 590 & 2.033 & 530 & 0.010 \\
\hline B.1.2 & None & None & One & Axi. & Yes & 0.901 & 4.94 & 590 & 4.991 & 530 & 0.009 \\
\hline B.2.1 & Aft & Fwd & One & Axi. & Yes & 0.900 & 4.94 & 590 & 2.027 & 530 & 0.021 \\
\hline $\mathrm{B} .2 .2$ & $\overline{\mathrm{Aft}}$ & Fwd & One & Axi. & Yes & 0.900 & 4.94 & 590 & 2.997 & 530 & 0.021 \\
\hline B.2.3 & Aft & Fwd & One & Axi. & Yes & 0.900 & 4.94 & 590 & 5.033 & 530 & 0.021 \\
\hline B.3.1 & Mid & $\overline{\mathrm{Mid}}$ & Two & Axi. & Yes & 0.901 & 4.94 & 590 & 3.407 & 530 & -0.004 \\
\hline B.3.2 & Mid & Aft & Two & Axi. & Yes & 0.899 & 4.94 & 590 & 3.400 & 530 & -0.004 \\
\hline B.3.3 & Mid & Fwd & Two & Axi. & Yes & 0.899 & 4.94 & 590 & 3.402 & 530 & 0.031 \\
\hline
\end{tabular}

\begin{tabular}{|c|c|c|c|c|}
\hline B.1.1 & $C_{D P N}$ & $C_{D P A}$ & $C_{D P T}$ & $C_{D T T}$ \\
\hline Experiment $\cdot \cdot \cdot \cdot \cdot$ & -.0015 & 0.0291 & $0.0276 \pm 0.01$ & $0.0500 \pm 0.005$ \\
PAB3D-V10.... & -.0209 & 0.0260 & 0.0051 & 0.0389 \\
PAB3D-Mod.t.e. $\cdot . \cdot$ & .0006 & 0.0281 & 0.0287 & 0.0654 \\
\hline
\end{tabular}

\begin{tabular}{|c|c|c|c|c|}
\hline B.1.2 & $C_{D P N}$ & $C_{D P A}$ & $C_{D P T}$ & \multicolumn{1}{c|}{$C_{D T T}$} \\
\hline Experiment $\cdot \cdot \cdot \cdot$ & -.0096 & 0.0296 & $0.0191 \pm 0.01$ & $0.0423 \pm 0.005$ \\
PAB3D-V10 . . . . . & -.0330 & 0.0253 & -0.0077 & 0.0259 \\
\hline
\end{tabular}

likely caused by the linear k- $\epsilon$ turbulence model formulation in which there is no separation of the normal stresses. The actual axisymmetric jet flow does have some anisotropy in the viscous stresses that were not modeled in Version 10 of the code. Also, no components of normal stress were predicted close to the plume centerline $\left(z / D_{m}<0.15\right)$. This discrepancy is also probably caused by the simplification of the turbulence modeling. The thickness of the shear layer calculated is fairly close to that of the data. The Reynold's stress $\overline{u^{\prime} w^{t}} / u_{\infty}^{2}$, figure 9 , is predicted fairly well with only minor differences with changes in the t.e. geometry. The centerline distributions of the axial velocity, shown in figure 10 indicate that the modified t.e. caused a higher gradient in axial velocity from $0 .<x / D_{m}<0.5$. This trend was observed previously in the Mach contours of figures 5 and 6 . The larger internal lip radius of the modified trailing edge altered the point of the jet shear flow origin. This can be seen by the slight shift in the velocity curves at $x / D_{m}=0$. This slight streamwise shift in addition to the steeper velocity gradients made an accurate comparison of the radial flow surveys difficult.

\section{Langley B Test Cases}

Photographs of the Langley test cases B.2 and B.3 installed in the Langley 16-Foot Transonic Tunnel are shown in figures 11 and 12 respectively. Test case B.1 is identical to test case B.2 with the vertical and horizontal tails removed. Table 1 identifies the empannage arrangements and flow conditions for these three test cases.

\section{Test Cases B.1 and B.2}

Axisymmetric, single-engine afterbody experimental data with tails off (B.1) and tails on (B.2) were obtained using an air-powered, cold jet simulation system in the 16-Foot Transonic Tunnel at the Langley Research Center (see figure 11). All comparisons were made for data at $M=0.9$.
Experimental/computational comparisons were made for the tail-off and staggered-tail configurations $N P R=2$ (B.1.1 and B.2.1) and $N P R=5$ (B.1.2 and B.2.3). The interactive program Gridgen was used to generate a vertical half-plane symmetric five block grid for the configuration with tails. A single-cell wide version of the grid was used for the B.1 (no tails) configurations. A tabulation of the experimental and predicted pressure drag and total drag (pressure + skin friction) for B.1 is shown in the tables above. These drag coefficients have been normalized by the maximum crosssectional area of the body.

$$
\text { - }
$$

B.1.1 - Figures 13 and 14 show comparisons of theory with experimental data for the single jet, axisymmetric geometry. In general the surface static pressure distributions compared well with the data except for the trailing edge portion of the nozzle where the code over-predicted the stagnation at the trailing edge (solid line). This pressure coefficient mis-match is due in part to the approximation made in the nozzle-boattail geometry which modeled the trailing edge as a sharp point rather than the combination blunt base/open cavity of the actual model. This conclusion was reached as a result of further calculations performed by a summer research associate at Langley, Kristina Alexander, after the initial study was completed. Several variations on modeling the trailing edge region of the nozzle were attempted. The most realistic geometric description involved modeling the individual blunt bases of the outer afterbody (0.010 in.) and inner nozzle ( 0.025 in.) with the metric break cavity that occurred between the two shells. The total trailing edge thickness was approximately $0.101 \mathrm{in}$. thick. Computational results on this more realistic trailing edge geometry are shown in figures 13 (dashed line) and 14 (solid symbols). The lower recovery pressure shown in figure 13 for the realistic trailing edge increased the predicted drag on the nozzle (see tabulated data above) and improved the comparisons with experimental pressure drag coefficients shown

in

le

di

th

di

a.

$\phi$

v

.

F 
in figure 14. Additionally, the 2-equation linear k- $\epsilon$ turbulence model formulation of Jones \& Launder typically predicts static pressure levels in the vicinity of a nozzle trailing edge that are too high. Improved turbulence modeling has the potential of correcting the trailing edge pressure recovery discrepancy further.

B.2.1 - A comparison between the computed external static pressure coefficients and experimental data at several angular stations for the B.2.1 (staggered tails, $N P R=$ 2.027 ) case is shown in figures 15,16 , and 17 . The row $\phi=0$ is along the top centerline of the model in line with the vertical tail. The first $C_{p}$ rise (around $x / l=0.64$ ) is caused by pressure stagnation at the leading edge of the vertical tail. Further downstream, there is a region where the pressure distribution was affected by the flow over the vertical and the horizontal tail surfaces as well as the afterbody and nozzle surfaces. The computed $C_{p}$ values were in general good agreement with the measured data. However, the local minimum near $x / l=0.9$ was not captured in the numerical simulation. The computed pressure recovery downstream of $x / l=0.9$ followed the measured data closely until near the end of the nozzle surface. The computed pressure coefficient was approximately 10 percent higher than the experimental data at the position of the last pressure orifice. This discrepancy is probably caused by the same factors as discussed for the the over-prediction noted for the B.1.1 case. Qualitatively, comparisons at other rows of pressure measurement positions were similar or better than those shown in figure 10.

A comparison of the integrated afterbody and nozzle pressure drag coefficients with measured values are shown in figure 18. The following table provides a tabulation of the results. These drag coefficients have been normalized by the maximum cross-sectional area of the body.

\begin{tabular}{|c|c|c|c|}
\hline B.2.1 & $C_{D P N}$ & $C_{D P A}$ & $C_{D T T}$ \\
\hline Experi & -.0130 & 0.0410 & $0.1055 \pm 0.005$ \\
\hline
\end{tabular}

The computed pressure drag coefficient of the afterbody compared well with the measured value. The comparison of the pressure drag coefficient on the nozzle external surface was less favorable. As one may expect from the over prediction of the pressure recovery in this region, the pressure recovery benefit was also over-predicted.

\section{Test Case B.3}

The twin-engine afterbody, cold-jet test data were obtained in the NASA-Langley Research Center 16-Foot Transonic Tunnel, (see figure 12). Surface static pressure data were measured on the afterbody and tail surfaces. Nozzle pressure drag and total aft-end (includes afterbody, nozzle, and tail surfaces) drag are available at $M=0.9$ at $N P R=3.4$. The program Gridgen was used to generate the computational meshes. Only the mid-vertical tail (B.3.1) and the forward-vertical tail (B.3.3) configurations (see Table 1) were gridded and approximately 1.5 million grid points in 11 blocks were used to describe the model including the nose, wing, afterbody, and nozzle internal geometry.
The computed and measured pressure coefficients along a row at the top-center position above the left side engine are compared in figure 19. Both the mid-position (B.3.1) and the forward position (B.3.3) vertical tail leading edge positions are clearly indicated in the $C_{p}$ distributions $(x / l=0.782$ and 0.727 , respectively). In the region between the vertical tails, the flow expanded steadily to attain supersonic speeds in both cases with $C_{p}$ values as low as -0.56 . The flow expansion was terminated by a shock ahead of the trailing edge of the vertical tail in both cases. An unusual detail at $x / l=0.95$, in the form of a small dip in $C_{p}$ was captured in these flow solutions. This feature is thought to be caused by a slope discontinuity between the afterbody and the nozzle. Pressure recovery on the nozzle compared well with data.

The pressure coefficient comparisons along the model top surface centerline on the interfairing between the engines and nozzles are shown in figure 20 . The last data point at $x / l=0.95$ was near the trailing edge of the interfairing between the twin engines. The computed flow expansions and shock positions were similar to those shown in figure 19 . This result indicates that the flow expansion and shock near the vertical tail surfaces (and noted for figure 19) carried all the way across the entire afterbody upper surface between the twin vertical tails. Similar to the B.2.1 case, there are several other pressure orifice rows where $C_{p}$ distribution comparisons between computation and experimental data were made for test case B.3, but are not shown in the current paper. The agreements were similar in quality and in some cases better than those shown in figures 19 and 20 .

Some detail of the B.3.3 flow solution on the inboard side of the nozzle is shown by the computed particle traces in figure 21. Although the pressure distribution on the inboard side of the nozzle was similar to that over the top of the nozzle, a mild flow separation had occurred, according to the computations. The computed isobars in the tail region of the B.3.3 case are shown in figure 22. The pressure gradients at the leading edge of the vertical tail, the flow expansion and shock between the vertical tails surfaces, and pressure recovery on the nozzle surface are clearly shown in this figure.

Measured and computed nozzle pressure drag and total aft-end drag for twin-engine test cases B.3.1 and B.3.3 are tabulated in the following table. These drag coefficients have been normalized by the wing reference area of the model.

\begin{tabular}{|c|l|l|}
\hline Conf. & \multicolumn{1}{|c|}{$C_{D P N}$} & \multicolumn{1}{|c|}{$C_{D T T}$} \\
\hline Exp.B.3.1 $\ldots .$. & -.0020 & $0.0084 \pm 0.0005$ \\
PAB3D-V10 $\ldots .$. & -.0020 & 0.0106 \\
Exp.B.3.3 $\ldots . .$. & -.0022 & $0.0078 \pm 0.0005$ \\
PAB3D-V10... & -.0022 & 0.0102 \\
\hline
\end{tabular}

The measured nozzle pressure drag values were predicted well by the computation but the total aft-end drag values (includes skin friction predictions) were not well predicted. However, it is interesting to note that the predicted drag increment between B.3.1 and B.3.3 was well predicted. This would indicate that this level of grid density and the V.10 simplified $k-\epsilon$ turbulence model is not able to predict 
absolute drag but may be useful in predicting effect on drag of incremental configuration changes.

\section{Conclusions}

The results of this investigation are summarized as follows:

1

1. Jet plume characteristics were matched closely by computational methods. The effect of trailing edge geometry modeling was discernible, but in general did not alter the comparisons with data. Limitations in linear turbulence models will preclude prediction of any anisotropy in the plume turbulence.

2. External static pressure distributions on single-engine and twin-engine configurations were predicted quite well matching both level and location of the experimental data features. Improvements in the single-engine, axisymmetric-nozzle trailing edge pressure recovery was obtained by a more accurate grid model of the geometry with emphasis in the trailing edge region. Improved turbulence modeling potentially could further improve the predicted pressure recovery.

3. Compared to experiment, integrated pressure drag predictions were typically better than total drag predictions. This level of grid resolution and simplified linear turbulence model may preclude accurate total force predictions.

\section{References}

1. Report of the Working Group on Aerodynamics of Aircraft Afterbody, AGARD AR-226, June 1986.

2. Abdol-Hamid, K. S.; Carlson, J. R.; and Lakshmanan, B.: Application of Navier-Stokes Code PAB3D to Attached and Separated Flows for Use With $k-\epsilon$ Turbulence Model, NASA TP-3480, 1994.

3. Abdol-Hamid, K. S.: A Multiblock/Multizone Code (PAB3D-v2) for the Three-Dimensional Navier-Stokes Equations: Preliminary Applications, NASA CR-182032, October 1990.

4. Sarkar, S,; Erlebacher, G.; Hussaini, M. Y.; and Kriess, H. O.: The Analysis and Modeling of Dilatational Terms in Compressible Turbulence, J. Fluid Mech., Vol. 227, June 1991, pp. 473-495.

5. Launder, B. E. and Sharma, B. I.: Application of the Energy Dissipation Model of Turbulence to the Calculation of Flow Near a Spinning Disk. Letters in Heat and Mass Transfer, Vol. 1, 1974, pp. 131-138.

6. Carlson, John R.: A Nozzle Internal Performance Prediction Method, NASA TP-3221, October 1992.

7. Abdol-Hamid, K. S.; Carlson, J. R.; and Pao, S. P.: Calculation of Turbulent Flows Using Mesh Sequencing and Conservative Patch Algorithm. AIAA 95-2336, 31st. Jt. Propulsion Conference, July 1995. 


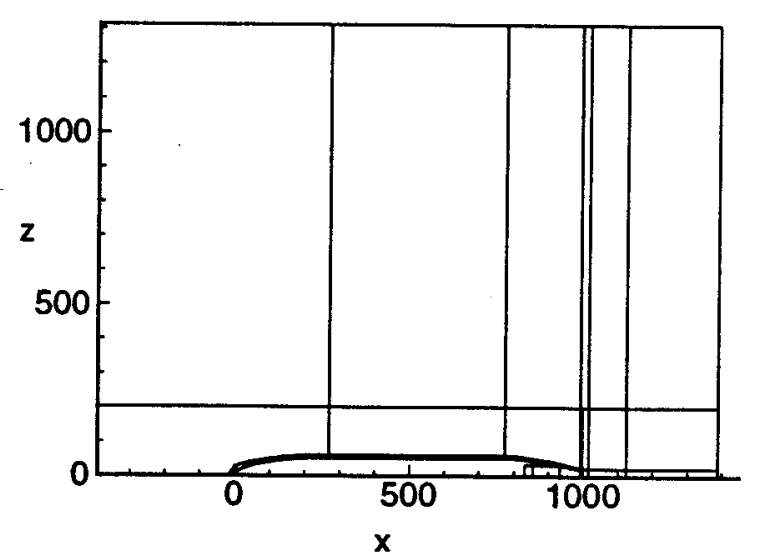

Figure 1.- Full grid block boundaries for A.2.

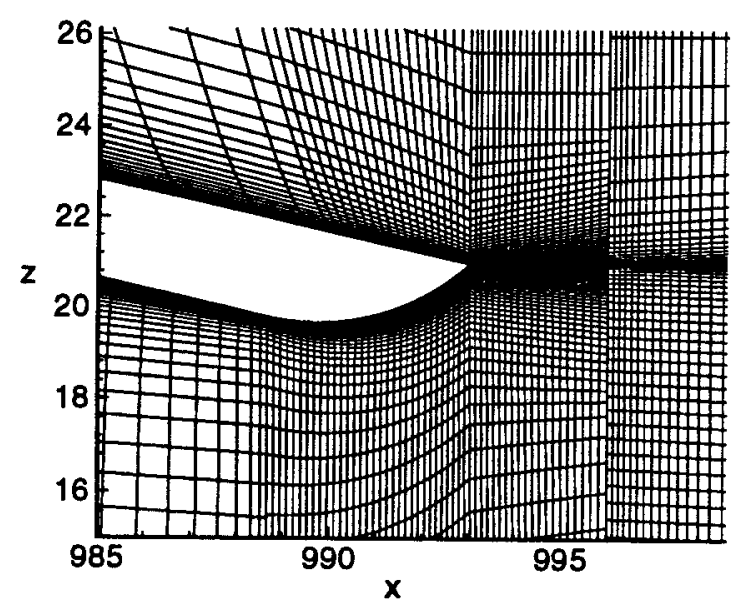

Figure 3.- Detail of modified trailing edge.

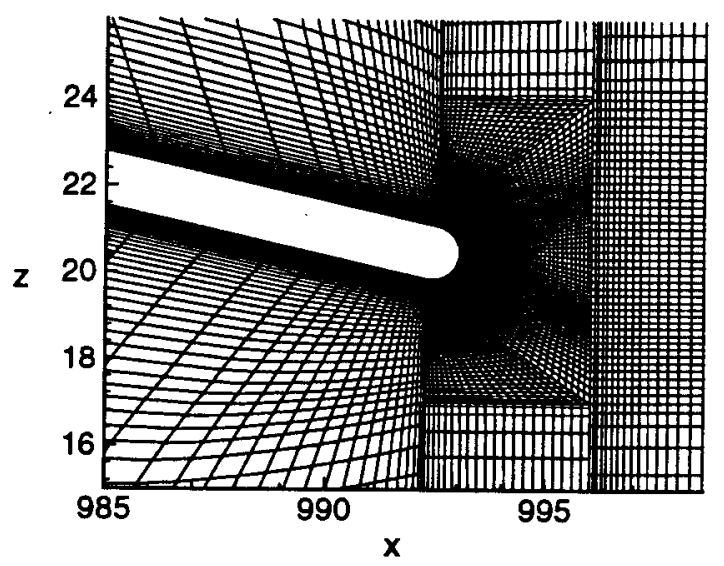

Figure 2.- Detail of original trailing edge.

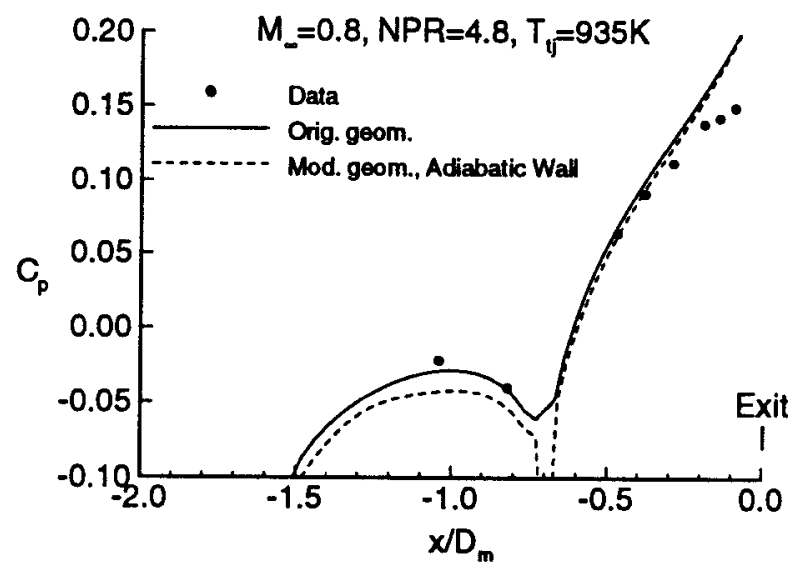

Figure 4.- Effect of t.e. geometry on surface static pressure distributions.

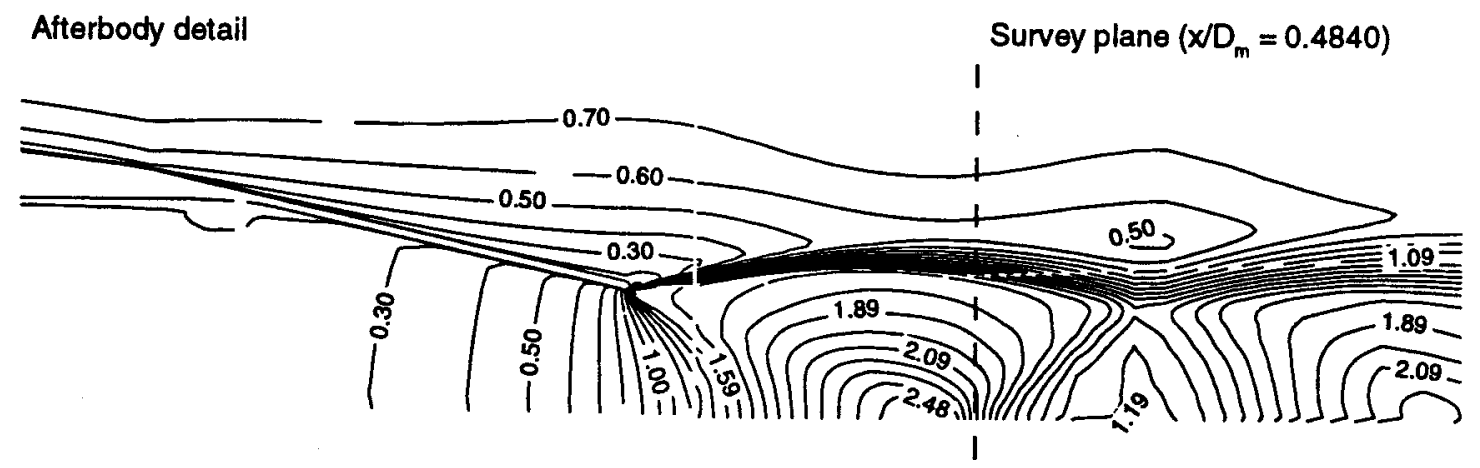

Figure 5.-Predicted Mach contours for ONERA A.2.2, original t.e.. 


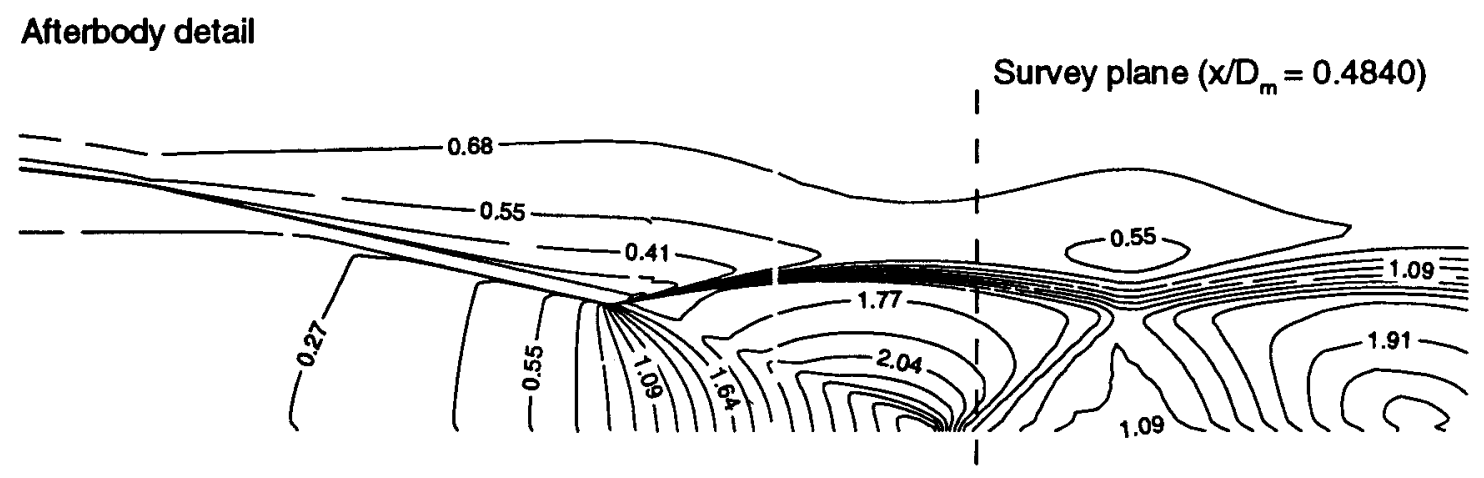

Figure 6.- Predicted Mach contours for ONERA A.2.2, modified t.e..
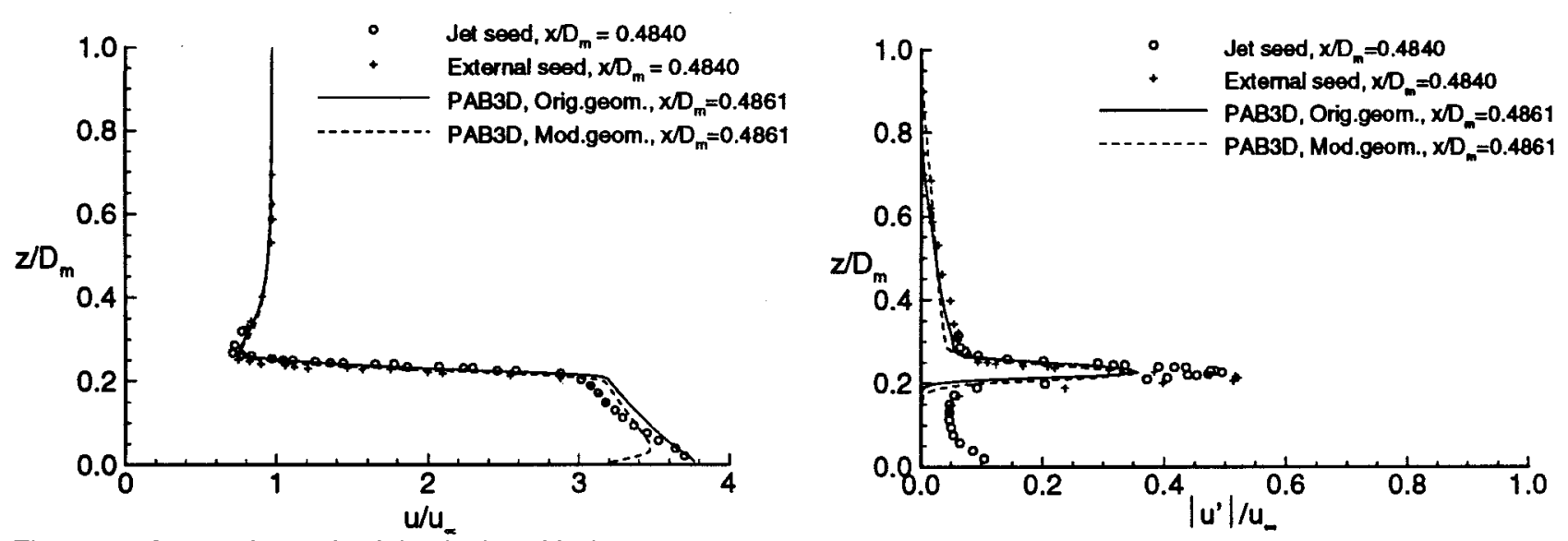

Figure 7.- Comparison of axial velocity with data,

A.2.2.

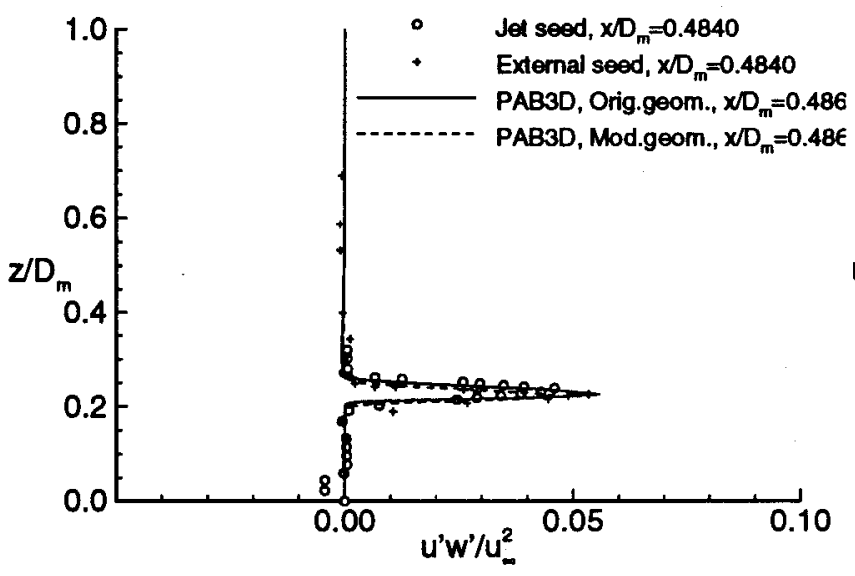

Figure 9.- Comparison of u'w' Reynold's stresses with data, A.2.2.
Figure 8.- Comparison of axial normal stresses with data, A.2.2.

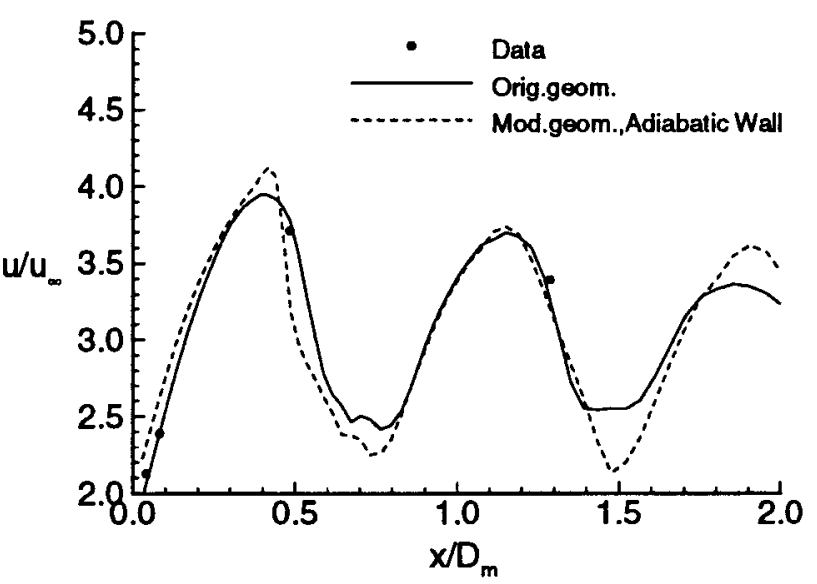

Figure 10.- Effect of t.e. geometry on centerline axial velocity. 


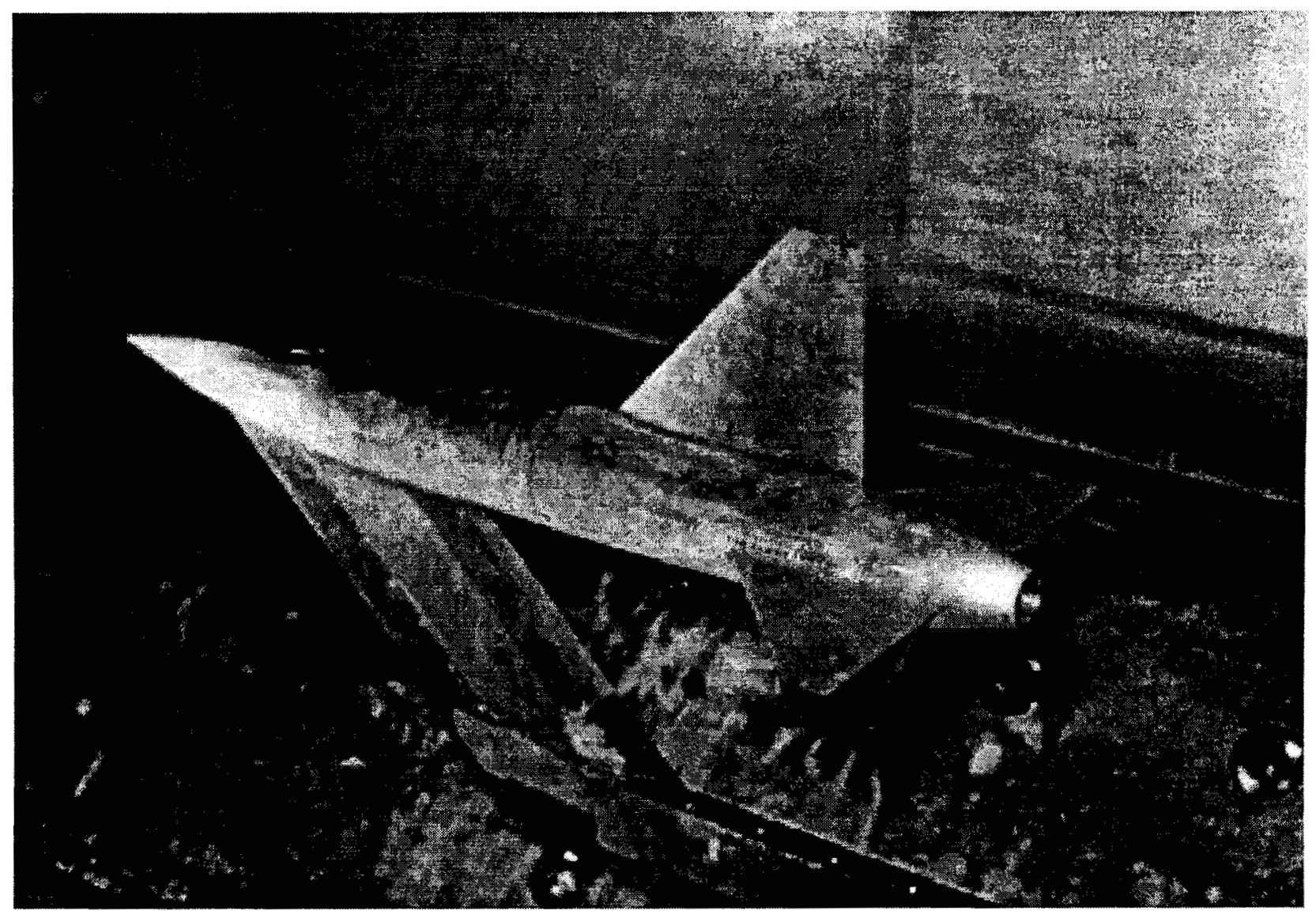

Figure 11.- Photograph of B.2 in the Langley 16-Foot Transonic Tunnel.

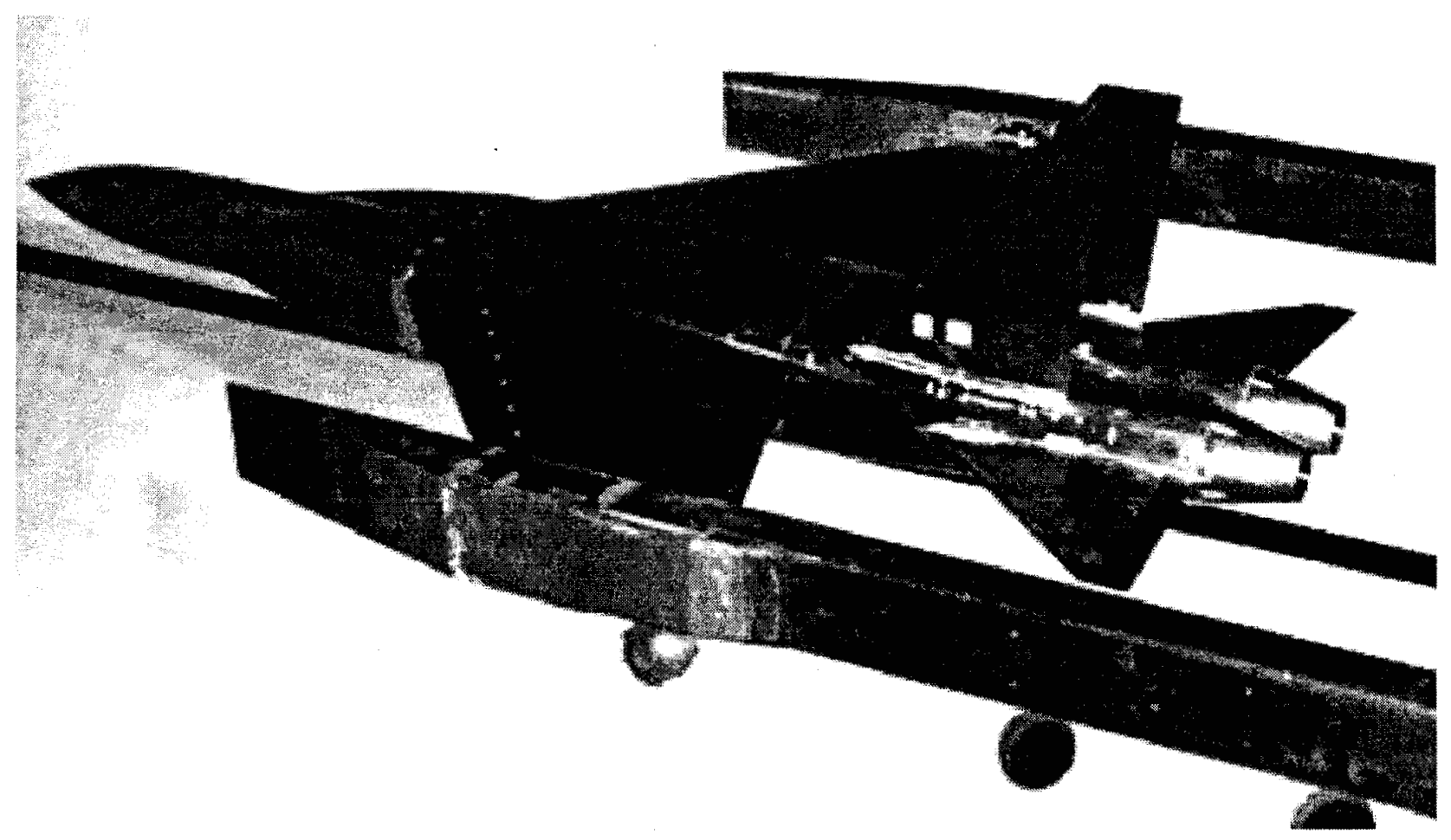

Figure 12.- Photograph of B.3 in the Langley 16-Foot Transonic Tunnel. 


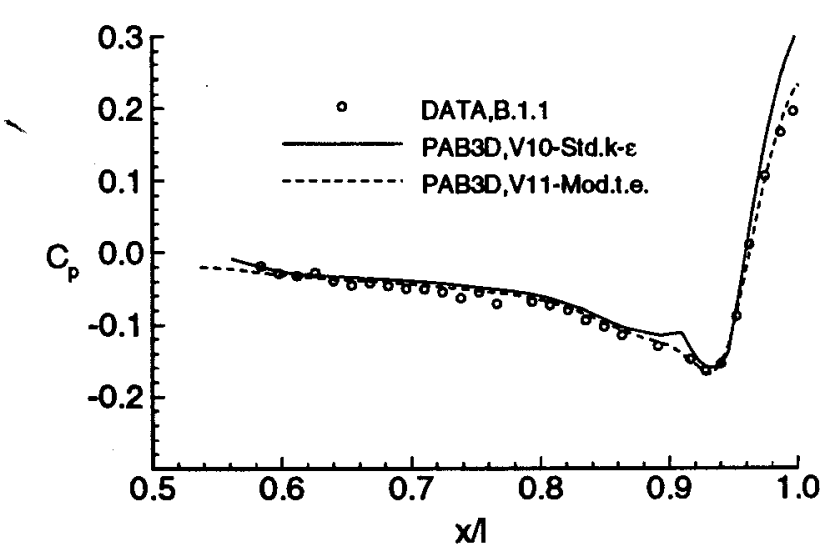

Figure 13.-Comparison of experimental and calculated pressure coefficient, $\mathrm{M}=0.90, \mathrm{NPR}=2.033$.

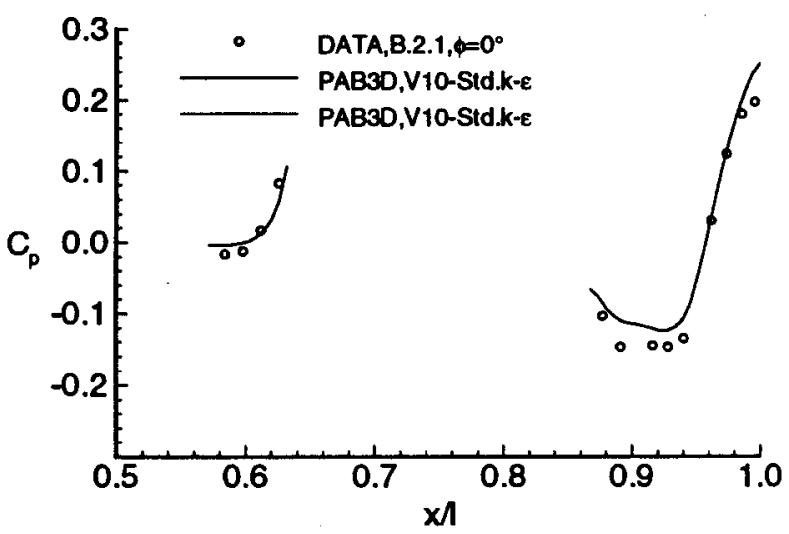

Figure 15.- Comparison of experimental and calculated pressure coefficient, $\mathrm{M}=0.90, \mathrm{NPR}=2.027$.

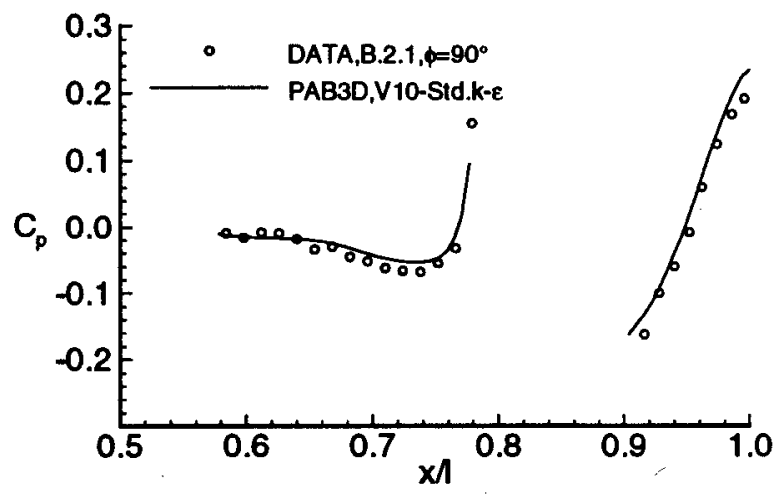

Figure 17.- Comparison of experimental and calculated pressure coefficient, $M=0.90, N P R=2.027$.

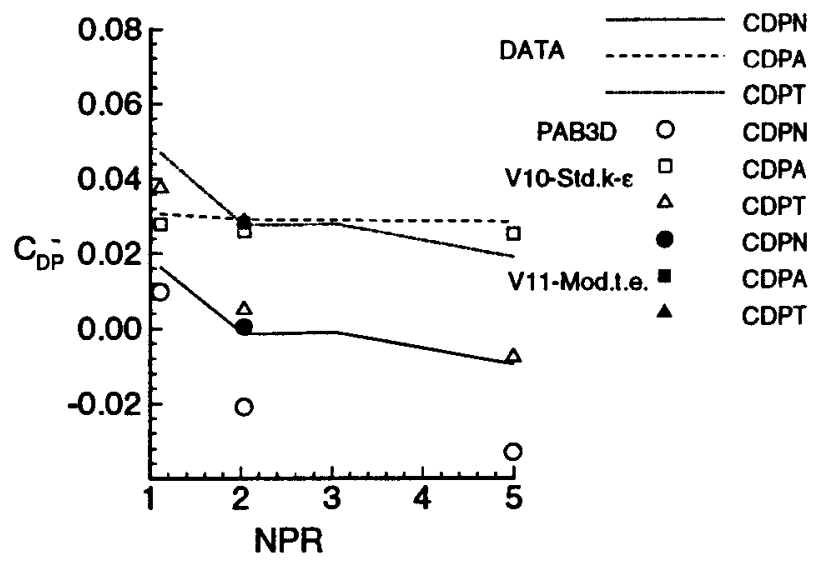

Figure 14.-Comparison of experimental and calculated pressure drag, $B .1, M=0.90$.

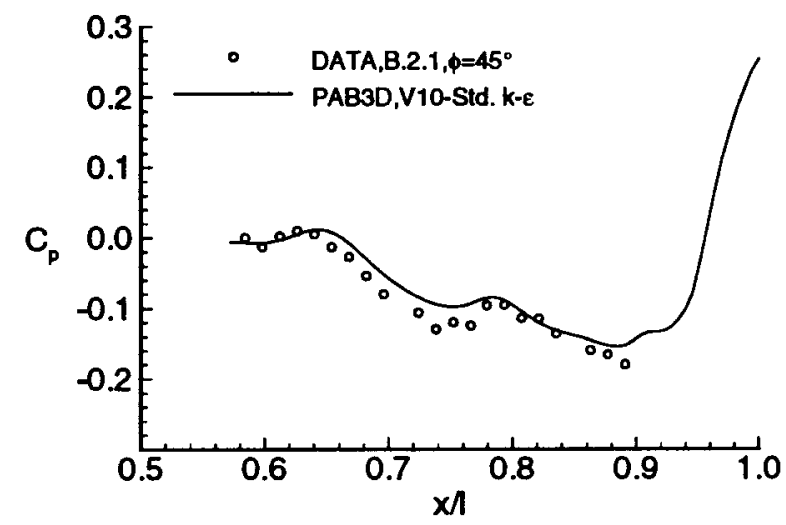

Figure 16.- Comparison of experimental and calculated pressure coefficient, $M=0.90, N P R=2.027$.

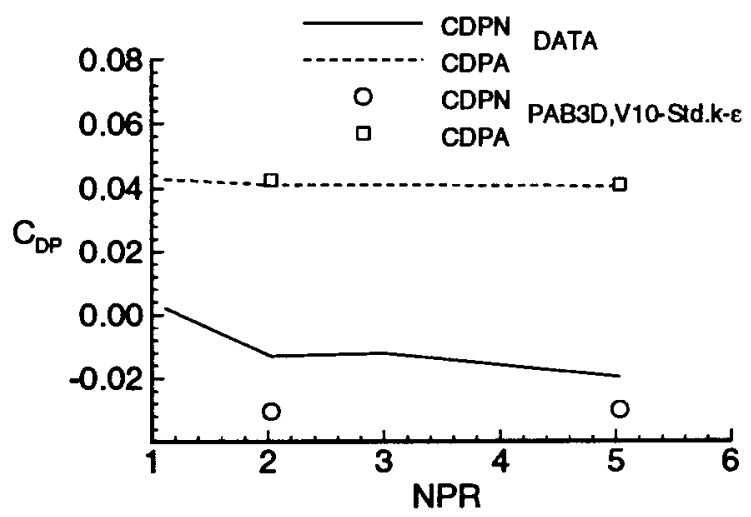

Figure 18.- Comparison of experimental and calculated pressure drag, B.2. 


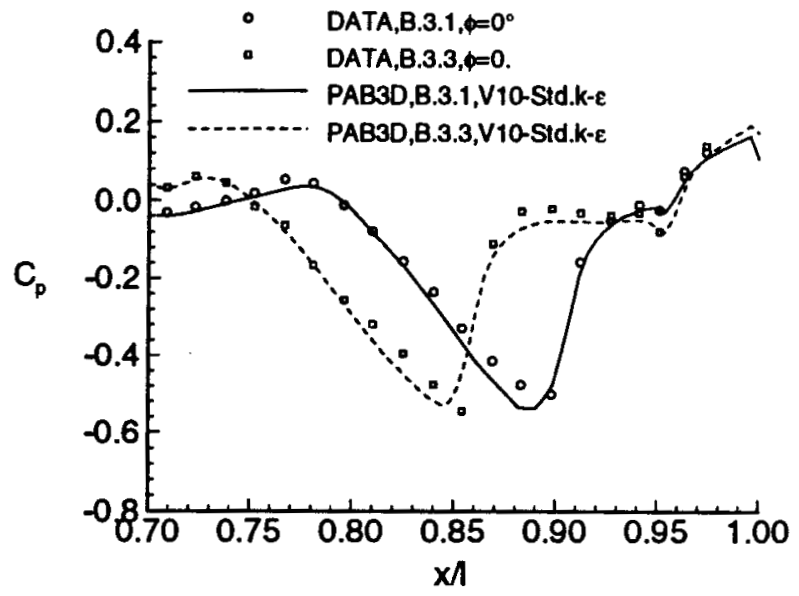

Figure 19.- Comparison of experimental and calculated pressure coefficients, B.3.

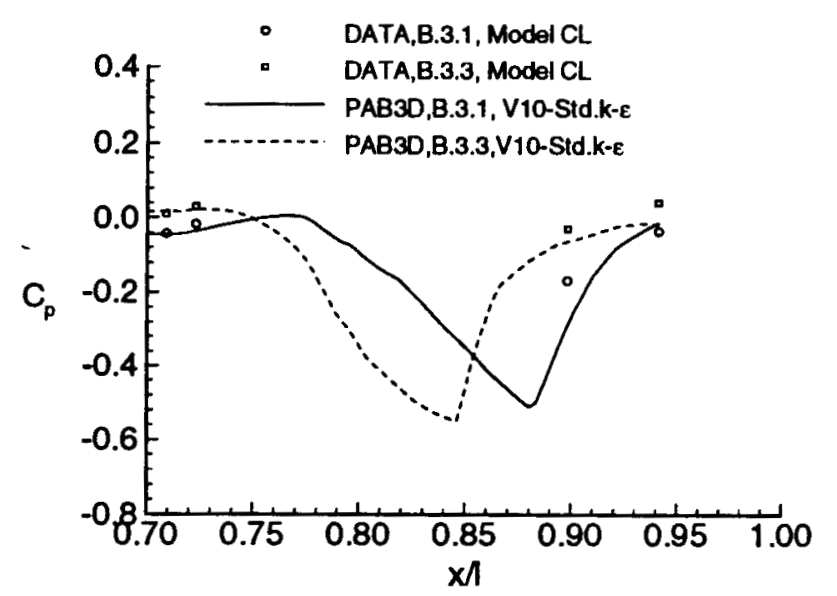

Figure 20.-Comparison of experimental and calculated pressure coefficients, B.3.

Afterbody detail

Tails omitted

$M=0.9$

$\mathrm{NPR}=3.4$

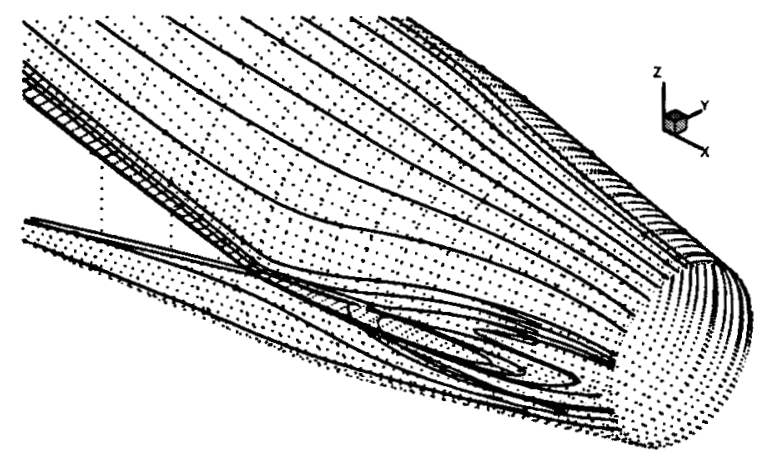

Figure 21.-Particle trace on nozzle external surface, B.3.3.

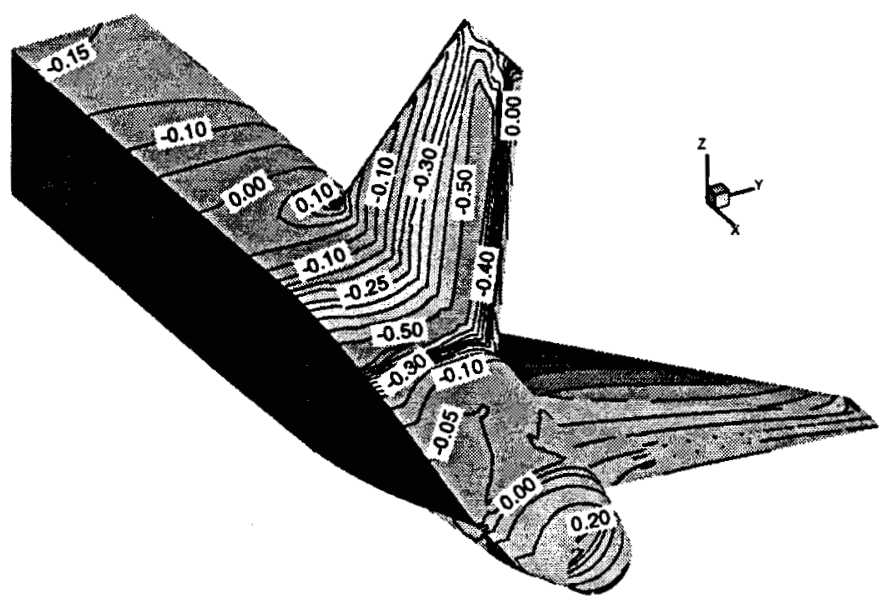

Figure 22.-Predicted surface static pressure contours, B.3.3.

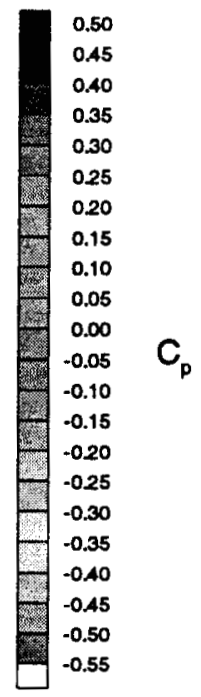

11

American Institute of Aeronautics and Astronautics 